\title{
Medication errors in infants at home
}

Shalini Ojha, Imti Choonara

Academic Unit of Child Health, University of Nottingham, Derbyshire Children's Hospital, Derby, UK

The study by Solanki and colleagues involved interviewing 166 parents/grandparents at home regarding the medications that had been prescribed at discharge to their infants, by the hospital staff [1]. As part of the study, the parents were also asked to demonstrate how much medicine they would give. With this methodology, Solanki et al. estimated that two out of three of the infants in their study would have experienced medication errors at home. This is an alarmingly high proportion of medication errors. Fortunately, none of the infants experienced significant harm. The authors have suggested that this high rate may be due to lack of parental education and inadequate predischarge counselling. The study was performed in Pondicherry in India. It would be wrong, however, to dismiss the relevance of their findings when considering the possibility of medication errors among neonates discharged from centres from high income countries, such as the U.K.

\section{How frequent are medication errors?}

Medication errors are often not recognised, and even when detected, often underreported. It is difficult to state the prevalence of medication errors in paediatric patients because of the wide variation in the definition of medication errors used by different investigators [2]. A literature review published in 2007 identified 11 studies looking at medication errors in the neonatal intensive care unit (NICU) [2]. The most common type of error in most studies was dosing errors. Dosing errors are a potentially major problem in neonates and infants for several reasons. Firstly, ten-fold errors are more likely to occur in a neonate or young infant where a small volume of drug is due to be administered. Additionally, immaturity of drug metabolism may also predispose to greater toxicity rending even small errors to be potentially more damaging than it would in the proportionately larger children or adults.

There have been relatively few studies of medication errors in infants or children at home. An American study of children with malignancies identified 72 medication errors occurring at home in a group of 92 children [3]. They identified a $70 \%$ risk of errors occurring in their patients, which is remarkably similar to the findings by Solanki.

\section{Clinical significance}

Most prospective studies of medication errors or adverse drug reactions do not detect significant toxicity due to the small number of patients involved in the study. In order to detect significant toxicity, one needs to use a different approach. A novel approach was to look at newspaper reports involving medicines. Over an eight year period (1993-2000) in the UK, there were at least 29 deaths in paediatric patients due to medication errors [4]. Nine of these 29 deaths involved neonates and the most frequent type of medication error involved an incorrect dose.

The American study of children with malignancies identified four medication errors that resulted in significant patient injury in their group of 92 patients [3]. Additionally, there were two life threatening errors which did not result in injury. These findings therefore suggest that significant 
medication errors in infants at home may be more common than generally assumed. The clinical significance of medication errors in paediatric patients at home remains unknown and can only be answered by large pharmacovigilance studies involving families and reaching out into homes. Infants are at greater risk of drug toxicity for a variety of reasons. Drug elimination pathways (both hepatic and renal) are not fully developed in neonates and young infants, particularly those born prematurely [5]. Over the years such physiological immaturities have come into focus due to now well known drug toxicities such as, the grey baby syndrome (chloramphenicol), kernicterus (sulphonamides), hepatotoxicity (sodium valproate), calcium precipitation (ceftriaxone and calcium containing solutions) and Reye's syndrome (salicylate) [5].

Some groups of medications are more likely to be associated with clinically significant toxicity following a medication error [6]. Cytotoxics, antiepileptics, opiates and insulin all may be associated with significant toxicity following dosing errors. One therefore needs to take greater care with such medications. Most infants in the U.K. are discharged home on medications such as multivitamins and iron supplements, similar to the participants of Solanki et al. and these medications are believed to be relatively innocuous even if minor medications errors occur. However, as highlighted by Solanki et al., large volume errors are common and it is possible that infants may be subjected to unnecessary risks if parents are not appropriately empowered to deliver medications at home. In addition, it is worth considering that with increasing trends or early discharges from both postnatal wards and neonatal units, more and more infants are likely to be discharged on short and long term medications that will need to be delivered at home by the parents. In this scenario the findings of Solanki et al. is a worthwhile reminder of the need to extend pharmacovigilance to include delivery of drugs at home.

\section{Minimising medication errors at home}

It is not possible to eliminate every single medication error. It is however possible to reduce the likelihood of the occurrence of a significant medication error. Firstly, the importance of giving appropriate information to parents when their infant is discharged from hospital should be recognised. Discharging a patient is usually left to the most junior doctor whose education, therefore, must include the importance of educating parents. Written information needs to be comprehensible to the patient. It is of concern that in the study in Pondicherry that prescriptions were written in English, a language that most of the parents were unable to read. This too might be relevant in the current multicultural milieu in the U.K. Greater care needs to be given when discharging infants on potentially toxic medications such as anti-epileptic drugs. Additionally, additional safeguards such as ensuring that community health professionals prescribe and dispense formulations of the same strength as that prescribed on discharged from hospitals. The responsibility for minimising medication errors to infants at home should rest primarily on health professionals, not parents and our jobs should include empowering parents to care for their infant.

\section{References}

1. Solanki R, Mondal N, Mahalakshmy T, Vishnu B. Medication errors by caregivers at home in neonates discharged from the NICU. Arch Dis Child in press.

2. Chedoe I, Molendijk HA, Dittrich STAM, et al. Incidence and nature of medication errors in neonatal intensive care with strategies to improve safety. Drug Safety 2007; 30: 503-513. 
3. Walsh KE, Roblin DW, Weingart SN, et al. Medication errors in the home: a multisite study of children with cancer. Pediatrics 2013; 131: e1405-e1413.

4. Cousins D, Clarkson A, Conroy S, Choonara I. Medication errors in children - an eight year review using press reports. Paed Perinat Drug Ther 2002; 5: 52-58.

5. Sammons HM, Choonara I. Learning lessons from adverse drug reactions in children. Children (Basel) 2016. doi:10.3390/children3010001

6. Choonara I. How to harm children in hospital - A guide for junior doctors. Paed Perinat Drug Ther 1999; 3: 34-5 